\title{
Avaliação da influência do etanol sobre o grau de volatilização BTEX em solos impactados por derrames de gasolina/etanol
}

\section{Evaluation of the ethanol influence over the volatilization grade of BTEX in soil impacted by gasoline/ethanol spills}

\author{
Alexandra Rodrigues Finotti \\ Engenheira Civil. Doutora em Recursos Hídricos e Saneamento Ambiental. Professora do Centro de Ciências Exatas e Tecnologia. Pesquisadora do Instituto \\ de Saneamento Ambiental da Universidade de Caxias do Sul (UCS)
}

Cláudia Echevenguá Teixeira

Bióloga. Doutora em Engenharia Ambiental. Professora do Centro de Ciências Exatas e Tecnologia. Pesquisadora do Instituto de Saneamento Ambiental da UCS e do Instituto de Pesquisas Tecnológicas (IPT)

Franciele Fedrizzi

Acadêmica de graduação em Engenharia Ambiental na UCS. Bolsista de iniciação científica da UCS

Joice Calgliari

Engenheira Ambiental do Instituto de Saneamento Ambiental da UCS

Irajá do Nascimento Filho

Licenciado em Química. Doutor em Química Ambiental. Professor do Centro de Ciências Exatas e Tecnologia e pesquisador do Instituto de Saneamento Ambiental da UCS

\section{Resumo}

O principal objetivo deste trabalho foi a avaliação quantitativa da influência do etanol sobre a volatilização de BTEX (benzeno, tolueno, etilbenzeno e xilenos) em mistura de gasolina e etanol anidro 25\% (v/v) em colunas experimentais, que simularam solos contaminados com gasolina pura e gasolina/etanol. Todos os BTEX apresentaram expressivo aumento das taxas de volatilização na coluna contendo a mistura gasolina/etanol. Porém, em termos percentuais, o maior e menor aumento nas taxas de volatilização foi observado para tolueno e benzeno, respectivamente. Em amostras de controle, com o percentual de etanol variando entre 0 e 25\%, não foi observado aumento no grau de volatilização do etilbenzeno, enquanto que o grau de volatilização dos xilenos foi reduzido. Estes resultados sugerem que, além de forças de interação intermoleculares, efeitos de interação líquido/estrutura do solo podem estar exercendo importante papel na volatilização dos BTEX.

Palavras-chave: etanol; BTEX; cromatografia; GC/FID; volatilização; contaminação de solo.

\begin{abstract}
The main objective of this paper was the quantitative evaluation of the ethanol's influence about the volatilization of BTEX (benzene, toluene, ethylbenzene and xylenes) in a mixture of gasoline and anhydrous ethanol 25\% (v/v) in experimental columns that simulated soil contamination with gasoline/ethanol. All the BTEX presented expressive increase of volatilization rates in the gasoline-ethanol column. However, in terms of percentage, the highest and lowest volatilization grades were observed for toluene and benzene, respectively. In batch tests (control samples), with mixtures of gasoline ethanol with 0 and $25 \%$ ( $/ \mathrm{v}$ ) in ethanol, no increase of the volatilization grade was observed for ethylbenzene and the volatilization grade for xylenes was reduced. Matrix effects seem to be, besides the intermolecular interaction forces, important contributions for the volatilization grade of BTEX in this kind of sample.
\end{abstract}

Keywords: ethanol; BTEX; chromatography; GC/FID; volatilization; soil contamination. 


\section{Introdução}

Combustíveis fósseis, como gasolina e óleo diesel, sempre formaram a principal matriz energética para a movimentação de veículos à combustão interna. Entretanto, apesar da aceitação do uso destes tipos de combustíveis e da convivência dos postos de abastecimento com áreas urbanas, a crise do petróleo dos anos 1970, o fato de que estes produtos são constituídos por algumas substâncias altamente poluentes e perigosas (FINOTTI, 2003) e os recentes estudos sobre o aquecimento global, levaram a sociedade moderna a procurar formas de geração de energia menos dispendiosas, com menores taxas de emissões de poluentes e menos tóxicas. O etanol e o biodiesel são dois exemplos do que hoje se convencionou chamar de biocombustíveis.

Aproximadamente 90\% da composição do petróleo é uma mistura complexa de hidrocarbonetos, o restante é constituído por compostos orgânicos sulfurados, nitrogenados, oxigenados e organometálicos. A gasolina é constituída de uma mistura de hidrocarbonetos voláteis, cujos componentes majoritários são cadeias ramificadas de parafinas, cicloparafinas e compostos aromáticos, incluindo benzeno, tolueno, etilbenzeno e xileno (BTEX).

A contaminação de solos e águas subterrâneas com BTEX é, normalmente, acompanhada pela liberação de vapores que migram da superfície para o ar. Estes vapores quando inalados podem oferecer riscos à saúde humana e de outros animais (CHIARANDA, 2006). O benzeno é cancerígeno, podendo causar leucemia, vertigens e tremores. Além disso, o benzeno afeta o sistema nervoso central e, quando contamina os aquíferos, torna-os inúteis para consumo humano. Segundo a resolução do Conselho Nacional do Meio Ambiente

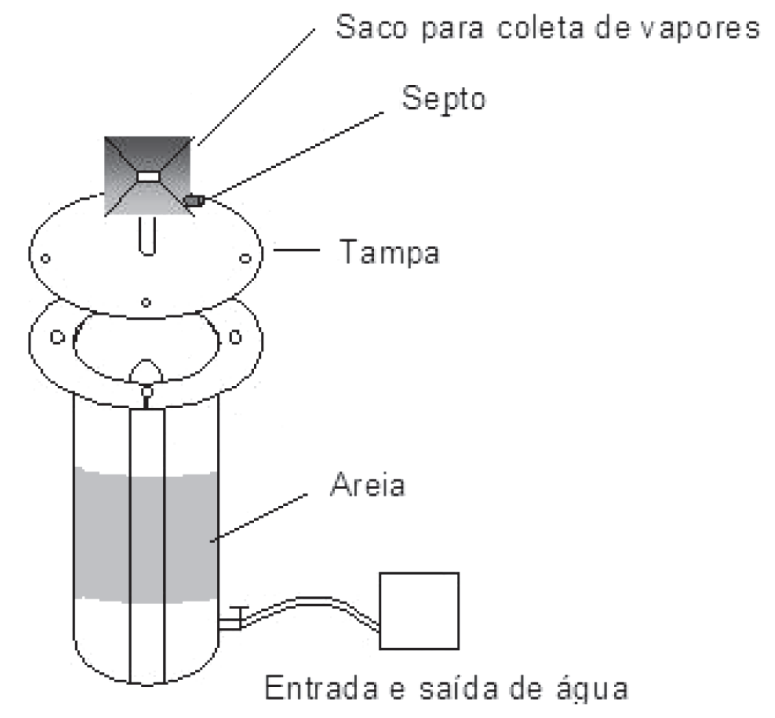

Figura 1 - Coluna experimental
(Conama) no 357/2005, a concentração máxima para o benzeno em águas doces é de $0,005 \mathrm{mg} / \mathrm{L}$. A inalação de altas concentrações dos vapores da gasolina, por exemplo, pode deprimir o sistema nervoso central, causando sensações de embriaguez, inconsciência e até mesmo levar à morte (ACGIH, 2001).

O Brasil é o país que adiciona a maior proporção de etanol na gasolina. Esta proporção tem variado entre 20 e 26\%. Apesar de trazer benefícios econômicos para o país, esta mistura modifica o comportamento físico e químico de vários compostos presentes na gasolina, o que pode representar efeitos negativos sobre os processos ambientais. À medida que aumenta a concentração do etanol misturado à gasolina, pode ocorrer um aumento da solubilidade dos hidrocarbonetos aromáticos na água subterrânea. Este processo é denominado efeito de cossolvência e provoca o aumento da magnitude da contaminação como consequência do aumento da concentração dos compostos orgânicos na água.

Neste contexto, o principal objetivo deste trabalho é determinar a influência do etanol em misturas gasolina/etanol, sobre as taxas de volatilização dos BTEX na gasolina. Foram utilizadas colunas acrílicas preenchidas com areia para simulação de solos atingidos por derrames de gasolina pura e gasolina/etanol. Também foram realizados ensaios em microcosmos para avaliar possíveis efeitos de interação líquido/estrutura do solo sobre a volatilização dos BTEX, utilizando gasolina pura e gasolina/etanol.

\section{Material e métodos}

\section{Colunas experimentais}

Para a simulação de solos contaminados, foram utilizadas duas colunas acrílicas de $1 \mathrm{~m}$ de altura e 0,3 m de diâmetro. As duas colunas foram montadas de forma a simular um aquífero com zona saturada, franja capilar e zona parcialmente saturada. Cada coluna foi preenchida até $90 \mathrm{~cm}$, com $115 \mathrm{~kg}$ areia de granulometria selecionada (diâmetro variando de 0,25 a 0,59 mm). A areia, previamente caracterizada por meio de ensaio de retenção e granulometria, apresenta pressão de borbulhamento de 0,20 mca, saturação residual de $19 \%$, porosidade de $37,7 \%$, coeficiente de não uniformidade de 1,90 e coeficiente de curvatura de 1,4. A umidade inicial da areia é muito baixa, com um valor equivalente a $0,012 \%$. As colunas foram embebidas com água destilada e, posteriormente, drenadas de forma a deixar uma lâmina de água de $30 \mathrm{~cm}$ no fundo da coluna, com o objetivo de formar a franja capilar no meio poroso. Finalmente, foi feita a contaminação com $1,15 \mathrm{~L}$ de gasolina pura (coluna 1) e o mesmo volume de uma mistura gasolina/etanol anidro (25\% (v/v) em etanol), na coluna 2. O objetivo destes procedimentos foi formar uma fase livre em ambas as colunas. Após o fechamento, foram acoplados sacos coletores de gases no topo de cada uma das colunas experimentais, representadas esquematicamente na Figura 1. 
$\mathrm{O}$ acrílico tem sido frequentemente utilizado para o estudo de compostos orgânicos, tais como biofiltração de BTEX (ABUMAIZAR et al, 1998), biodisponibilidade e naftaleno (BURGOS et al, 1999). Baek et al (2003) estudaram a adsorção de benzeno em areia e avaliaram a influência de colunas confeccionadas em vidro e acrílico sobre os resultados. Segundo os autores, o uso de acrílico não afetou, de forma importante, os resultados de transporte e adsorção do benzeno.

Os vapores das colunas experimentais foram coletados semanalmente durante 263 dias, com o auxílio de uma seringa Gastight (Hamilton), volume máximo de $1 \mathrm{~mL}$, à temperatura ambiente. Após cada coleta, os sacos para gases foram esvaziados com o auxílio de uma seringa de $250 \mathrm{~mL}$ para provocar uma nova situação de equilíbrio e avaliar as novas proporções dos BTEX na fase de vapor.

\section{Amostras controladas}

Para avaliar possíveis efeitos de interação líquido/estrutura do solo sobre a volatilização dos BTEX foram preparados microcosmos em dois frascos de vidro contendo $20 \mathrm{~mL}$ de gasolina pura e uma mistura de gasolina e etanol (25\% (v/v) em etanol). Os frascos de vidro, com capacidade para $50 \mathrm{~mL}$ e contendo as amostras controladas foram fechados com septo de borracha butílica, selados com anéis de alumínio e mantidos em repouso, durante 24 horas à temperatura ambiente. Após este tempo, um volume de 0,3 mL da fase de vapor de cada uma das amostras controladas foi analisado por cromatografia à gás, em triplicata. As amostragens foram realizadas utilizando-se seringas Gastight - Hamilton nas mesmas condições descritas anteriormente. Após cada coleta, foram retirados $20 \mathrm{~mL}$ da fase de vapor de cada amostra controlada, com a finalidade de forçar um novo equilíbrio entre as fases líquida e de vapor.

\section{Análise instrumental}

A análise instrumental dos BTEX presente nos vapores das colunas experimentais e das amostras controladas foi realizada por injeção no modo splitless, em triplicata, de 0,3 mL das amostras em um cromatógrafo à gás Perkin Elmer, modelo Autosystem XL, equipado com um detector por ionização de chama - GC/FID. Foi utilizada uma coluna capilar Elite ( $30 \mathrm{~m}$ versus $0,25 \mathrm{~mm}$ d.i. versus $0,25 \mu \mathrm{m}$ de espessura de filme), com a seguinte programação de temperatura: $40^{\circ} \mathrm{C} / 10 \mathrm{minu}-$ tos, rampa de aquecimento de $5^{\circ} \mathrm{C} /$ minuto até $220^{\circ} \mathrm{C} / 0$ minuto. O gás de arraste utilizado a um fluxo de $1 \mathrm{~mL} /$ minuto foi hélio.

\section{Resultados e discussão}

\section{Colunas experimentais}

Os volumes de vapores nas colunas 1 e 2, após o encerramento do período de coleta, foram de 160 e $220 \mathrm{~mL}$, respectivamente. Foi observado que os meses mais quentes resultaram em uma produção maior de gás, muito provavelmente pelo aumento na pressão de vapor dos BTEX. Os limites de detecção para os BTEX, determinados a

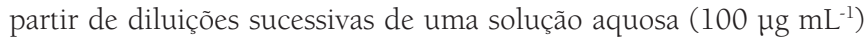
foram de 0,4 $\mu \mathrm{g} \mathrm{mL}^{-1}$, para benzeno, tolueno e etilbenzeno e 0,3 $\mu \mathrm{g}$ $\mathrm{mL}^{-1}$, para os xilenos.

Nas colunas experimentais, todos os BTEX apresentaram aumento das concentrações na fase de vapor, porém este aumento foi muito mais acentuado na coluna 2 (mistura gasolina/etanol).

A mistura de etanol a hidrocarbonetos da gasolina forma azeótropos de mínima, os quais possuem temperatura de ebulição menor que a dos componentes isolados (CATALUÑA; SILVA, 2006). Esse efeito tem como consequência o aumento da pressão de vapor da mistura e, como resultado, o favorecimento da volatilização dos BTEX. Entretanto, este efeito ocorre em extensões diferentes para cada BTEX, como pode ser observado na Figura 2, em função das diferenças de suas propriedades físico-químicas.

O enorme aumento na volatilização dos BTEX em função da presença do etanol está evidenciado na Tabela 1, na qual se observa que, em termos relativos, tolueno e xilenos apresentaram os maiores aumentos de concentração na fase de vapor.

Um das propriedades físico-químicas que pode ter influenciado no aumento das concentrações dos BTEX na fase de vapor das colunas experimentais é a polaridade ou momento de dipolo (COMPANION, 1964). Quando uma molécula altamente polarizada (momento de dipolo>>zero, como o etanol, por exemplo) é colocada nas vizinhanças de outras moléculas de baixa polaridade (como os BTEX), ocorre a deformação da nuvem eletrônica desta última. Esta deformação torna a molécula de baixa polaridade momentaneamente polarizada. Neste momento podem ocorrer interações do tipo London, dipolo ou van der Waals, que desaparecem quando a molécula polar é afastada. As moléculas de etilbenzeno e dos xilenos têm polaridade suficientemente alta (Tabela 2) para promover fortes interações intermoleculares com as moléculas de etanol. Sendo assim, o grau de volatilização destes hidrocarbonetos tende a ser reduzido na mistura gasolina/etanol.

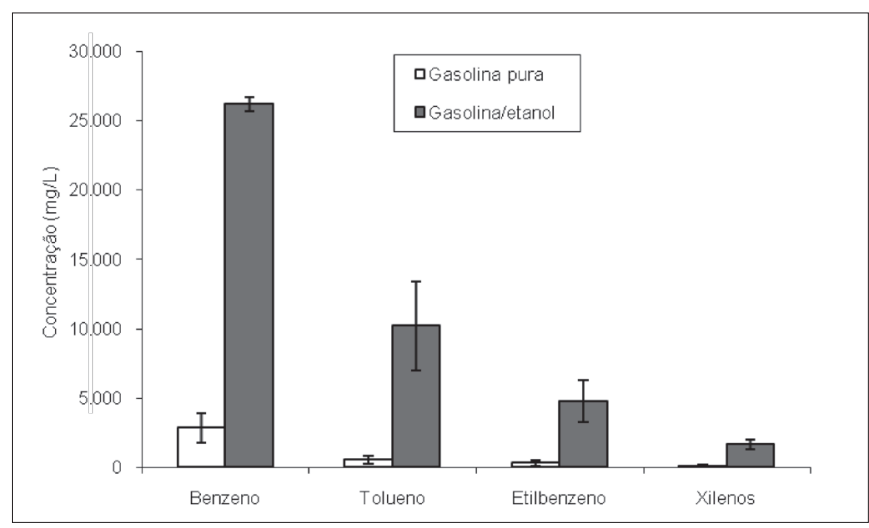

Figura 2 - Grau de volatilização dos BTEX nas colunas contaminadas com gasolina pura e gasolina/etanol 
Tabela 1 - Evolução das quantidades de BTEX nas fases de vapor das colunas experimentais contendo gasolina pura e gasolina/etanol

\begin{tabular}{|c|c|c|c|c|}
\hline & & \multicolumn{3}{|c|}{ Tempo (dias) } \\
\hline & & 27 & 160 & 263 \\
\hline Amostra & Composto & Concentração médiac & Concentração média & Concentração média \\
\hline \multirow[t]{4}{*}{ GPa } & Benzeno & $14,76( \pm 5,15)$ & $1.786,17( \pm 182,55)$ & $2.838,49( \pm 1.041,16)$ \\
\hline & Tolueno & $N D^{d}$ & $742,23( \pm 290,88)$ & $528,15( \pm 284,51)$ \\
\hline & Etilbenzeno & $1,62( \pm 1,37)$ & $161,35( \pm 8,79)$ & $302,48( \pm 197,49)$ \\
\hline & Xilenos & ND & $63,19( \pm 8,45)$ & $103,90( \pm 42,11)$ \\
\hline \multirow[t]{4}{*}{$\mathrm{MIST}^{\mathrm{b}}$} & Benzeno & $7,22( \pm 2,70)$ & $608,17( \pm 367,75)$ & $26.172,36( \pm 528,58)$ \\
\hline & Tolueno & ND & $707,41( \pm 344,15)$ & $10.183,47( \pm 3.189,46)$ \\
\hline & Etilbenzeno & ND & $61,68( \pm 25,80)$ & $4.767,25( \pm 1.492,15)$ \\
\hline & Xilenos & ND & $46,35( \pm 15,19)$ & $1.642,73( \pm 366,00)$ \\
\hline
\end{tabular}

${ }^{a}$ Coluna experimental contendo gasolina pura; ${ }^{b}$ Coluna experimental contendo a mistura gasolina/etanol (25\% (v/v) em etanol; ${ }^{\mathrm{c}} \mathrm{mg} \mathrm{L}-1$ ( $\mathrm{n}=3$ ); ${ }^{\mathrm{d} N a ̃ o}$ detectado ou abaixo do limite de detecção.

Tabela 2 - Algumas propriedades físico-químicas dos BTEX (LIDE, 1996)

\begin{tabular}{|c|c|c|c|c|c|}
\hline Composto & Momento de dipolo (D) & $\begin{array}{l}\text { Densidade } \\
\qquad\left(\mathrm{g} \mathrm{cm}^{-3}\right)\end{array}$ & $\begin{array}{c}\text { Solubilidade de água } \\
\qquad\left(\mathrm{mg} \mathrm{L}^{-1}\right)\end{array}$ & Pressão de vapor (atm) & 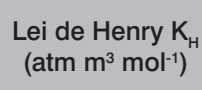 \\
\hline Etanol & 1,69 & 1,49 & $\infty$ & $0,58 \cdot 10^{-1}$ & $6,29.10^{-6}$ \\
\hline Benzeno & 0 & 0,87 & 175 & $1,25.10^{-1}$ & $8,30.10$ \\
\hline Tolueno & 0,37 & 0,86 & 535 & $3,70.10^{-2}$ & $6,37.10^{-3}$ \\
\hline o-xileno & 0,59 & 0,88 & 178 & $8,61 \cdot 10^{-3}$ & $5,10.10^{-3}$ \\
\hline m-xileno & 0,3 & 0,86 & 161 & $1,08.10^{-3}$ & $7,18.10^{-3}$ \\
\hline p-xileno & 0,02 & 0,1 & 162 & $1,15.10^{-3}$ & $6,90 \cdot 10^{-3}$ \\
\hline Etilbenzeno & 0,64 & 0,87 & 152 & $9,00.10^{-3}$ & $6,43.10^{-3}$ \\
\hline
\end{tabular}

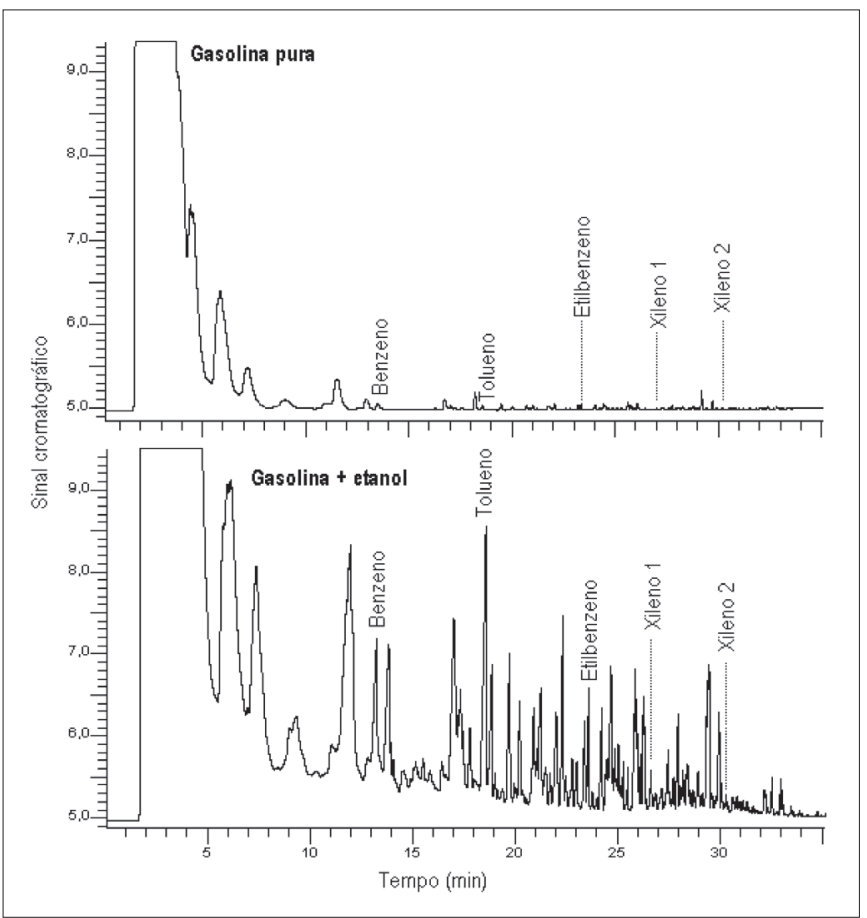

Figura 3 - Cromatogramas típicos das amostras de vapor das colunas contendo gasolina pura e gasolina/etanol

O aumento da taxa de volatilização do tolueno na mistura gasolina/etanol também pode ser explicado pelo fenômeno das interações intermoleculares. Este composto apresenta menor polaridade (LIDE,
1996) que etilbenzeno e xilenos (Tabela 2) e, desta forma, sofre menor influência da presença das moléculas de etanol, podendo ocupar o espaço na fase de vapor, deixado pelas moléculas de etilbenzeno e xilenos. Corrêa e Arbilla (2007) identificaram o tolueno como o principal composto orgânico volátil na atmosfera do centro da cidade do Rio de Janeiro. Porém, o aumento de volatilização do benzeno não pode ser explicado pela teoria das interações intermoleculares, uma vez que este é um composto apolar e; portanto, sem possibilidade de desenvolver este tipo de interação com as moléculas de etanol.

A Figura 3 apresenta os cromatogramas típicos das amostras de vapor das colunas experimentais.

\section{Amostras controladas}

A Figura 4 apresenta a variação das concentrações dos BTEX nas amostras controladas.

Estes resultados contrastam amplamente com aqueles observados para as colunas experimentais, uma vez que o único BTEX a apresentar aumento de concentração na fase de vapor da mistura gasolina-etanol foi o tolueno. Sendo assim, é necessário admitir que, além das interações de dipolo, outros fatores devem influenciar no aumento da volatilização de BTEX, a partir de derrames de gasolina e etanol no solo. A própria volatilização do etanol é uma questão a ser considerada, pois se a sua concentração na fase líquida é reduzida por evaporação, as interações intermoleculares 
tornam-se muito mais fracas, o que favorece a volatilização dos BTEX. O trabalho publicado por Pumphrey et al (2000) traz suporte para esta hipótese. Estes autores realizaram experimentos com misturas de gasolina e vários álcoois, dentre eles o etanol, com o objetivo de avaliar a influência do teor de álcool na variação da pressão de vapor da mistura. No caso da mistura com etanol, verificou-se que a pressão de vapor parte de um valor de 64,63 kPa e aumenta, proporcionalmente com o aumento do teor de etanol, até a proporção de $10 \%$ (v/v) para depois se estabilizar em torno de $70 \mathrm{kPa}$, até a proporção de 25\% (v/v) em etanol. Portanto, é razoável propor-se que, acima ou abaixo de uma determinada concentração de etanol na gasolina, as interações intermoleculares exerçam pouca ou nenhuma influência sobre a volatilização de BTEX na gasolina.

Outro fenômeno que deve ocorrer nas colunas é a transferência do etanol para a água da coluna contaminada com gasolina e etanol, em função da hidrofilia deste composto. Como o solo apresenta água na região da franja capilar e na zona parcialmente saturada, a migração do etanol para a água deve ser processada. Finotti (2003) avaliou a influência da transferência do etanol para a água e sua interferência na variação da tensão interfacial do sistema gasolina-água-etanol sem a presença de solo, e verificou que esta transferência ocorre rapidamente naquela situação. Na presença do solo, esta velocidade deve ser alterada, de forma que o sistema acaba entrando em um novo estado de equilíbrio. Porém, estes temas estão sendo investigados em outros experimentos.

\section{Conclusões}

Os resultados obtidos até o momento sugerem que, além da concentração de etanol e de possíveis interações intermoleculares, outros fatores, tais como degradação microbiológica, foto-decomposição, dispersão vertical e granulometria das partículas do solo (fatores ambientais, ausentes nas amostras controladas) e/ou efeitos

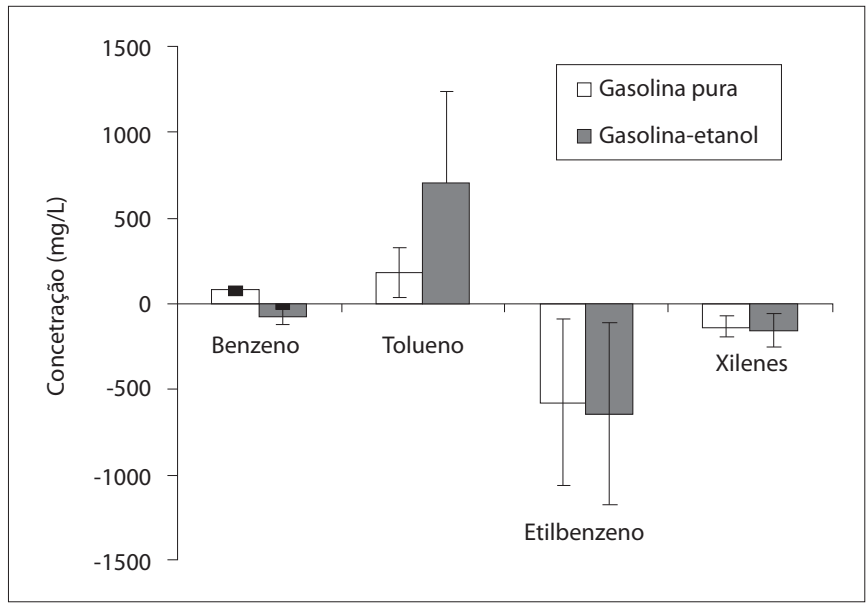

Figura 4 - Variação das concentrações médias dos BTEX na fase de vapor das amostras controladas

de interação líquido/estrutura do solo podem interferir na volatilização de BTEX de solos contaminados por derrames da mistura gasolina/etanol. Nos ensaios com amostras controladas, com exceção do tolueno, a adição de etanol à gasolina tem um importante efeito de redução da volatilização destes compostos, enquanto que nas colunas experimentais este efeito não é observado. Esses resultados sugerem que, além das características físico-químicas de cada BTEX, as condições ambientais devem exercer grande influência sobre a volatilização destes compostos e, por isto, devem ser estudadas com maior profundidade. Tanto nas colunas experimentais quanto nas amostras controladas foi observado o aumento da volatilização do tolueno, um composto químico reconhecidamente carcinogênico.

Obviamente estes são resultados preliminares e as próximas etapas deste trabalho deverão estar dirigidas justamente ao estudo das implicações de fatores ambientais ou efeitos de interação líquido/estrutura do solo, assim como a ampliação do período de amostragem sobre o grau de volatilização dos BTEX em misturas gasolina/etanol. Também serão realizados cálculos de balanço de massas nas colunas experimentais.

\section{Referências}

ABUMAIZAR, R.J.; KOCHER, W.; SMITH, E.H. Biofiltration of BTEX contaminated air streams using compost-activated carbon filter media. Journal of Hazardous Materials, v. 60, n. 2, p. 111-126, 1998.

ACGIH - American Conference of Governmental Industrial Hygienists. Documentation of Threshold Limit Values (TLV) for Chemical Substances and Physical Agents and Biological Exposure Indixes (BEI) for 2001. Cincinnati, OH: ACGIH, 2001.

BAEK, D.S.; KIM, S.B.; KIM, D.J. Irreversible sorption of benzene in sandy aquifer materials. Hydrological Processes, n. 17, p. 1239-1251, 2003. 
BURGOS, W.D., et al. Impact of soil-chemical interactions on the bioavailability of naphthalene and 1- naphtol. Water Research, v. 33, n. 18, p.3789-3795, 1999.

CATALUÑA, R.; SILVA, R. Desenvolvimento de um equipamento para avaliação do efeito do etanol na pressão de vapor e entalpia de vaporização em gasolinas automotivas. Química Nova, v. 29, n. 3, p. 580-585, 2006.

CHIARANDA, H.S. Volatilização dos compostos BTEX em ambientes subsuperficiais contaminados com gasolina e etanol: comparação entre concentrações medidas e simuladas. Dissertação (Mestrado em Engenharia Ambiental) - Universidade Federal de Santa Catarina, Florianópolis, 2006.

COMPANION, A.L. Chemical bonding. 3 ed. New York: McGraw-Hill, 1964. $140 \mathrm{p}$.
CORRÊA, S. M.; ARBILLA, G. A two-year monitoring program of aromatic hydrocarbons in Rio de Janeiro downtown area. Journal of the Brazilian Chemical Society, v. 18, n. 3, p.539-543, 2007.

FINOTTI, A.R. Estudos de fase livre em contaminações subsuperficiais com gasolina e etanol. Tese (Doutorado em Engenharia de Recursos Hídricos e Saneamento Ambiental) - Universidade Federal do Rio Grande do Sul, Brasil, 2003.

LIDE, D.R. Handbook of Chemistry and Physics. $76^{\text {th }}$ ed. Florida: CRC Press, 1996.

PUMPHREY, J.A.; BRAND, J.I.; SCHELLER, W.A. Vapour pressure measurements and predictions for alcohol - gasoline blends. Fuel, n. 79, p. $1405-1413,2000$ 